\title{
Avaliação do acesso e qualidade do atendimento odontológico na atenção básica de Patos-PB sob a perspectiva dos usuários
}

\author{
Evaluation of access and quality of dental care in basic care of Patos-PB from the perspective of \\ users \\ Evaluación del acceso y calidad de la atención dental en cuidados básicos de Patos-PB desde la \\ perspectiva de los usuarios
}

Recebido: 17/07/2021 | Revisado: 30/07/2021 | Aceito: 03/08/2021 | Publicado: 08/08/2021

\author{
Redson Alves Silvestre Junior \\ ORCID: https://orcid.org/0000-0001-7079-8757 \\ Universidade Federal de Campina Grande, Brasil \\ E-mail: redsonjunioralves@gmail.com \\ Maria Luísa de Assis Braga \\ ORCID: https://orcid.org/0000-0001-6563-4336 \\ Universidade Federal de Campina Grande, Brasil \\ E-mail: marialuisaabraga@hotmail.com \\ Tuanny Lopes Alves Silvestre Coura \\ ORCID: https://orcid.org/0000-0002-6122-9971 \\ Universidade Federal de Campina Grande, Brasil \\ E-mail: tuanny_silvestre@hotmail.com \\ Faldrécya de Sousa Queiroz Borges \\ ORCID: https://orcid.org/0000-0001-5960-1546 \\ Centro Universitário de Patos, Brasil \\ E-mail: faldrecya@gmail.com \\ Luciana Ellen Dantas Costa \\ ORCID: https://orcid.org/0000-0003-4476-7900 \\ Universidade Federal de Campina Grande, Brasil \\ E-mail: ellendantascosta@yahoo.com.br \\ Faldryene de Sousa Queiroz Feitosa \\ ORCID: https://orcid.org/0000-0001-8531-871X \\ Universidade Federal de Campina Grande, Brasil \\ E-mail: falqueiroz@ hotmail.com
}

\begin{abstract}
Resumo
O objeto da pesquisa foi avaliar o acesso e qualidade do atendimento odontológico na atenção básica do município de Patos-PB. Foi realizada uma entrevista com os usuários dos serviços odontológicos das Unidades Básicas de Saúde da Família (UBS) da cidade. Para coleta das informações foi utilizado um instrumento formulado a partir da agregação de questionários previamente validados: QASSaB, dados demográficos e dados sobre a autopercepção de saúde bucal e demanda aos serviços de saúde bucal. Os dados foram analisados pelo programa estatístico SPSS e o teste do Quiquadrado (IC 95\%) avaliou a associação entre as variáveis. Foram entrevistados 211 usuários, onde 61,6\% eram do sexo feminino e a maioria (27\%) tinha entre 31-40 anos de idade. 65,4\% declararam uma renda mensal familiar de 1-3 salários mínimos, 60,7\% nunca tiveram dor de dente e 70,1\% consideram sua saúde bucal boa. 38,9\% consideraram como "fácil" conseguir uma vaga para o tratamento odontológico na UBS, 32,2\% avaliou o tempo de espera como curto e $71,1 \%$ tiveram os problemas de saúde resolvidos. 47,4\% relataram estar "satisfeitos" com o tratamento odontológico e 53,1\% avaliaram como "confortável" a estrutura física das UBSs. A maioria relatou que raramente recebe orientações sobre saúde bucal $(60,7 \%)$ e os procedimentos de maior procura foram as exodontias $(28,8 \%)$ e restaurações (32,2\%). A localização da UBS apresentou relação significativa com a qualidade dos serviços $(\mathrm{p}=0,0015)$. Os usuários das UBSs de Patos-PB encontram-se relativamente satisfeitos com o atendimento odontológico realizado na atenção básica, tendo as suas queixas no geral prontamente atendidas pelos profissionais.
\end{abstract}

Palavras-chave: Odontologia; Avaliação em saúde; Atenção básica; Satisfação do usuário.

\begin{abstract}
The object of the research was to assess the access and quality of dental care in primary care in the city of Patos-PB. An interview was conducted with users of dental services of the Basic Family Health Units (UBS) in the city. To collect the information, an instrument formulated from the aggregation of previously validated questionnaires was used: QASSaB, demographic data and data on self-perception of oral health and demand for oral health services. Data were analyzed using the SPSS statistical program and the chi-square test $(95 \% \mathrm{CI})$ assessed the association between
\end{abstract}


variables. 211 users were interviewed, 61.6\% were female and the majority (27\%) were between 31-40 years old. $65.4 \%$ declared a monthly family income of 1-3 minimum wages, $60.7 \%$ never had a toothache and $70.1 \%$ considered their oral health to be good. $38.9 \%$ considered it "easy" to get a place for dental treatment at the UBS, $32.2 \%$ rated the waiting time as short and $71.1 \%$ had their health problems resolved. $47.4 \%$ reported being "satisfied" with the dental treatment and 53.1\% rated the physical structure of the UBSs as "comfortable". Most reported that they rarely receive guidance on oral health $(60.7 \%)$ and the most sought after procedures were extractions $(28.8 \%)$ and restorations (32.2\%). The location of the UBS showed a significant relationship with the quality of services $(\mathrm{p}=0.0015)$. Users of UBSs in Patos-PB are relatively satisfied with the dental care provided in primary care, and their complaints in general are promptly attended to by professionals.

Keywords: : Dentistry; Health assessment; Basic care; User satisfaction.

\section{Resumen}

El objeto de la investigación fue evaluar el acceso y la calidad de la atención odontológica en atención primaria en la ciudad de Patos-PB. Se realizó una entrevista a usuarios de los servicios odontológicos de las Unidades Básicas de Salud de la Familia (UBS) de la ciudad. Para la recolección de la información se utilizó un instrumento formulado a partir de la agregación de cuestionarios previamente validados: QASSaB, datos demográficos y datos de autopercepción de salud bucal y demanda de servicios de salud bucal. Los datos se analizaron mediante el programa estadístico SPSS y la prueba de chi-cuadrado (IC 95\%) evaluó la asociación entre variables. Se entrevistaron 211 usuarios, de los cuales el 61,6\% eran mujeres y la mayoría (27\%) tenían entre 31 y 40 años. El 65,4\% declaró un ingreso familiar mensual de 1-3 salarios mínimos, el 60,7\% nunca tuvo dolor de muelas y el 70,1\% consideró que su salud bucal era buena. El 38,9\% consideró "fácil" conseguir una plaza para tratamiento odontológico en la UBS, el $32,2 \%$ calificó el tiempo de espera como breve y el 71,1\% resolvió sus problemas de salud. El 47,4\% informó estar "satisfecho" con el tratamiento odontológico y el 53,1\% calificó la estructura física de las UBS como "cómoda". La mayoría informó que rara vez recibe orientación sobre salud bucal $(60,7 \%)$ y los procedimientos más solicitados fueron extracciones $(28,8 \%)$ y restauraciones $(32,2 \%)$. La ubicación de la UBS mostró una relación significativa con la calidad de los servicios $(\mathrm{p}=0,0015)$. Los usuarios de SBU en Patos-PB están relativamente satisfechos con la atención odontológica que se brinda en atención primaria, y sus quejas en general son atendidas con prontitud por profesionales.

Palabras clave: Odontología; Valoración de Salud; Atención primaria; Satisfacción del usuario.

\section{Introdução}

A Política Nacional de Humanização (HumanizaSUS), instituída no Brasil em 2003, veio para efetivar os princípios do SUS no cotidiano das práticas de atenção e gestão. Ela busca, na prática, a ampliação do acesso aos serviços de saúde, um atendimento acolhedor e resolutivo, estreitar o vínculo profissional/paciente, garantia dos direitos dos usuários e a valorização do trabalhador (Brasil, 2010).

Visando melhorar ainda mais o acesso e a qualidade do atendimento nos serviços públicos de saúde, em 19 de julho de 2011, foi criado pelo Ministério da Saúde o Programa Nacional de Melhoria e da Qualidade da Atenção Básica (PMAQ), cujo principal objetivo foi induzir a ampliação do acesso e a melhoria da qualidade da atenção básica, de maneira a permitir maior transparência e efetividade das ações governamentais direcionadas à Atenção Básica em Saúde (Brasil, 2012). Mas é sabido que há um grande distanciamento entre os princípios que norteiam o SUS e a realidade prática da saúde pública no Brasil.

Embora toda evolução nos últimos tempos no sistema de saúde pública do Brasil, percebe-se, ainda, que é um sistema com muitas lacunas. Backes et al. (2014) enfatizam, como principais falhas a dificuldade no acesso e a demora na realização do atendimento, resultado das inúmeras intervenções políticas, do desvio de verbas públicas e da falta de engrenagem sistêmica entre os diferentes setores. Reforça ainda que o SUS é uma rede perfeita, mas que, na prática, os fios/setores não se encontram e nem mesmo se cruzam, isto é, não conversam entre si para encontrar soluções conjuntas.

Bahia (2018) afirmou que ao longo dos últimos trinta anos, foi consolidada a ideia de que a resposta para os problemas de saúde no Brasil seria o SUS. No entanto, nesse mesmo período, cristalizaram-se visões negativas sobre a ineficiência, a má gerência de recursos, o descaso e a despersonalização do atendimento no setor público.

Tais fatores podem ser atribuídos a uma alta rotatividade de profissionais, dificultando a criação do vínculo 
profissional /paciente, a distribuição do número de profissionais por região e, principalmente, a qualificação técnica, ocasionando a precarização do serviço e dos trabalhadores, interferindo na motivação dos empregados, que evita estimular a desenvolver uma melhor assistência nas instituições do sistema (Moura, Pinto, \& Aragão, 2016).

A demora no agendamento e atendimento de consultas na atenção básica, o não atendimento da demanda espontânea e a falta de humanização no cuidado, constituem nos principais fatores para a baixa satisfação dos usuários com a Atenção Primária a Saúde (Gomide, Pinto, Bulgarelli, Santos, \& Gallardo, 2018).

Estudos sobre a satisfação do usuário têm sido enfocados na literatura como indicadores da qualidade da atenção, sendo a sua perspectiva avaliativa de grande importância, podendo levantar questões tanto em relação a estrutura quanto ao processo do cuidado da saúde. Eles oferecem suporte para a reorganização dos serviços, por meio de informações pertinentes e confiáveis, além de ser uma ferramenta de participação popular na gestão da saúde (Brandão, Giovanella, \& Campos, 2013; Perez et al. 2013).

Há um consenso para entender que a melhor forma de avaliar o sistema de saúde em vigor seria a partir das expectativas de quem é cuidado e sobre o que é importante para eles. Neste contexto, o presente trabalho objetivou avaliar o acesso e qualidade do atendimento odontológico na atenção básica do município de Patos-PB sob a perspectiva dos usuários do sistema.

\section{Metodologia}

O presente projeto foi submetido ao Comitê de Ética em Pesquisa do Hospital Universitário Alcides Carneiro (HUAC - UFCG), via Plataforma Brasil e aprovado sob protocolo n ${ }^{\circ}$ 3.620.849. Tratou-se de um estudo transversal, tipo inquérito, com abordagem quantitativa (Pereira et al., 2018). Realizou-se uma coleta de dados primários nas 40 Unidades Básicas de Saúde da Família (UBS) da zona urbana e 01 UBS da zona rural de Patos-PB.

A população foi composta pelos usuários dos serviços odontológicos da atenção básica da cidade de Patos-PB. A amostra foi por conveniência e constituída pelos usuários que se encontravam no local para atendimento no dia visitado, distribuída proporcionalmente entre as unidades de saúde, de acordo com a demanda atendida, com uma quantidade mínima de 5 entrevistas e máxima de 10, por UBS.

Foram incluídos na pesquisa os usuários dos serviços odontológicos das Unidades Básicas de Saúde (UBS) do município de Patos-PB, com idade mínima de 18 anos, que concordaram em participar da pesquisa e que assinaram um Termo de Consentimento Livre e Esclarecido (TCLE), e que no dia da entrevista encontravam-se na área de acolhimento da Unidade Básica de Saúde visitada, aguardando atendimento odontológico. Foram excluídos os menores de 18 anos, os portadores de necessidades especiais cognitivas, bem como aqueles cuja consulta odontológica seria a primeira na referida unidade.

O território da saúde no município de Patos-PB está dividido em quatro áreas denominadas Distrito GeoAdministrativo- (DGA), criado em 2009 pela Secretaria Municipal de Saúde, no intuito de descentralizar a administração da saúde e aproximá-la mais da população. Cada DGA é responsável por gerenciar uma média de 10 Unidades Básicas de Saúde (UBS).

Previamente a aplicação dos questionários, foi solicitada a Secretaria Municipal de Saúde uma listagem com o nome e endereço de todas as Unidades Básicas de Saúde, para garantir que todas tenham sido contempladas com a pesquisa. Ao chegar à unidade, foram esclarecidos aos participantes, os objetivos da pesquisa e a necessidade de responder a todas as questões, mas respeitando seu arbítrio caso optassem por deixar alguma sem resposta. Após os esclarecimentos, foi solicitada a assinatura do TCLE, assim como determina a Resolução no 466/12 de 12 de dezembro de 2012 do Conselho Nacional de Saúde que regulamenta pesquisas envolvendo seres humanos.

O TCLE foi elaborado em duas vias. A primeira ficou em posse do sujeito da pesquisa e outra arquivada e anexada ao 
formulário, de forma que apenas o pesquisador responsável tivesse acesso ao documento. Os usuários que se recusaram a participar, tiveram o direito de autonomia respeitado.

A coleta de dados se deu por meio de entrevistas individuais, aos usuários dos serviços odontológicos, realizadas pelo pesquisador, devidamente calibrado, nas 40 unidades de saúde da zona urbana do município de Patos, Paraíba, e 01 da zona rural. As entrevistas ocorreram no período de julho a dezembro de 2019, e foram distribuídas proporcionalmente entre as unidades de saúde, de acordo com a demanda atendida, com uma quantidade mínima de 5 entrevistas e máxima de 10, por UBS. Um estudo piloto foi realizado com 15 pacientes com o objetivo de treinar os entrevistadores e avaliar a adequação do instrumento de pesquisa.

Utilizou-se um instrumento formulado a partir da agregação de questionários previamente validados: QASSaB Questionário de Avaliação do Serviços de Saúde Bucal (Fernandes, 2002; Brunhauser, Magro, \& Neves, 2013; Magalhães, Oliveira, Goes, \& Figueiredo, 2015), dados demográficos (Brunhauser et al., 2013; Magalhães et al., 2015) e dados sobre a autopercepção de saúde bucal e demanda aos serviços de saúde bucal (IBGE/PNAD 2003; Magalhães et al., 2015). O QASSaB é composto por 24 questões e avalia diversas dimensões da qualidade de atendimento, como: disponibilidade dos serviços; resolutividade; ambiente físico do serviço-limpeza; relações humanas (relações com outros profissionais de saúde); relações humanas (relação dentista/paciente); qualidade técnico-científica dos equipamentos; eficiência; eficácia; equidade e aceitabilidade. Para análise da pontuação obtida através da aplicação do QASSaB, foi utilizado um escore final a partir da média aritmética dos escores de cada questionário, categorizado em três estratos: Insatisfeito/pouco satisfeito (de 0 a 2,0), satisfeito (de 2,0001 a 3,0) e muito satisfeito/totalmente satisfeito (de 3,0001 a 5,0), assim como proposto no estudo de Brunhauser et al. (2013).

Os dados foram processados com o auxílio do programa estatístico SPSS, versão 21.0. Para verificar associações entre a variável desfecho e as variáveis independentes, foram realizadas análises bivariadas empregando os testes qui-quadrado e exato de Fisher, com nível de significância de 5\% e intervalos de confiança de 95\% (IC95\%).

\section{Resultados}

\subsection{Perfil dos usuários que buscaram atendimento odontológico nas Unidades Básicas de Saúde}

Foram entrevistados 211 usuários, onde 61,6\% eram do sexo feminino e a maioria (27\%) tinha entre 31-40 anos de idade. No que diz respeito ao estado civil os entrevistados, $45,5 \%$ eram casados. A grande maioria dos participantes $(65,4 \%)$ declararam uma renda mensal familiar de 1-3 salários mínimos. Em relação ao grau de escolaridade, $39,8 \%$ tinham $2^{\circ}$ grau completo. Quanto à habitação 47,9\% dos participantes moram em casa própria e a grande maioria $(61,1 \%)$ é não beneficiário de programas sociais. Os dados relacionados ao perfil dos entrevistados encontram-se expostos na Tabela 1. 
Tabela 1. Perfil dos entrevistados. Patos, PB, 2020.

\section{Perfil dos entrevistados}

\section{Sexo}

Masculino

Feminino

\section{Localização da residência}

DGA 1

DGA 2

DGA 3

DGA 4

\section{Escolaridade}

$1^{\circ}$ grau incompleto $\mathrm{n}$

$1^{\circ}$ grau completo

$2^{\circ}$ grau incompleto

38

$2^{\circ}$ grau completo

Universidade completa

Pós-graduação

Não alfabetizado

Faixa etária

18 a 20 anos

21 a 30 anos

31 a 40 anos

41 a 50 anos

51 a 60 anos

\section{Renda mensal familiar}

Até 1 salário mínimo

1 - 3 salários

138

65,4

Mais de 3 salários

Sem informação

\section{Estado Civil}

Solteiro

Casado

Separado

Viúvo

\section{Beneficiário}

Sim

Não

Serviço que utiliza

Público 
Privado

\section{Habitação}

Alugada

Emprestada

Cedida

Própria

Financiada
12

5,7

32,7

3,8

12,3

$101 \quad 47,9$

2

Fonte: Autores.

\subsection{Saúde bucal dos entrevistados e avaliação do acesso e qualidade do atendimento:}

Os dados relacionados a saúde bucal dos entrevistados e avaliação do acesso e qualidade dos serviços encontram-se expostos na Tabela 2 .

Tabela 2. Dados relacionados a saúde bucal dos entrevistados e avaliação do acesso e qualidade dos serviços. Patos, PB, 2020.

Saúde bucal - acesso - qualidade

Já teve dor de dente

Sim

Não

Não lembra

Sem informação

\section{Como classifica sua saúde}

Excelente

Boa

Ruim

Sem informação

Motivo da procura

Exodontia

Restauração

Raspagem / limpeza

Consulta

Dor

Outros

\section{Vaga para o tratamento}

Muito difícil

Difícil

\section{$\%$}

34,1

60,7

4,7

0,5

$1 \quad 0,5$

148

9,0

43

70,

Nem difícil nem fácil
68

30

46

\section{8}

14

28,8

32,2

15,2

14,2

3,8

6,6

13,7

15,2

21,8 
Fácil

Muito fácil

Intervalo entre as consultas

Sem informação

Muito Longo

Longo

Nem curto nem longo

Curto

Muito curto

Tempo de espera

Sem informação

Muito longo

Longo

Nem longo nem curto

Curto

Muito curto

\section{Resolução do problema}

Sem informação

Não resolvido

Mal resolvido

+ ou - resolvido

Resolvido

Muito bem resolvido

\section{Satisfação com a aparência dos dentes}

Sem informação

Totalmente satisfeito

Muito satisfeito

Satisfeito

Um pouco satisfeito

Insatisfeito

\section{Satisfação com o tratamento}

Sem informação

Insatisfeito

Um pouco satisfeito

Satisfeito

Muito satisfeito

Totalmente satisfeito 


\subsection{Avaliação da estrutura física das UBS e equipe de saúde}

Os dados relacionados a avaliação da estrutura física das UBS e equipe de saúde encontram-se expostos na Tabela 3.

Tabela 3. Dados relacionados a avaliação da estrutura física das UBS e equipe de saúde. Patos, PB, 2020.

\section{Estrutura física e equipe}

\section{Limpeza da recepção}

Péssima

Ruim

Regular

Boa

Excelente

\section{Limpeza do consultório odontológico}

Sem informação

Ruim

Regular

Boa

Excelente

Limpeza do banheiro

Péssima

Ruim

Regular

Boa

Excelente

Sem informação

\section{Conforto}

Desconfortável

Pouco confortável

Confortável

Muito confortável

Totalmente confortável

Atenção da equipe de saúde

Ruim

Regular

Boa

Excelente

\section{Atenção do dentista}

Péssima

Sem informação
3

10

40

$\mathrm{n}$

$\%$

2

0,9

5

2,4

23

10,9

128

60,7

53

25,1

2

1,4

0,9

18

8,5

140

66,4

48

22,7

7

3,3

4

1,9

36

17,1

84

39,8

14

6,6

66

31,3

112

4,7

19

48

53,1

22,7

1

0,5

0,5

31

14,7

116

55

63

29,9

2 
Regular

Boa

Excelente

\section{Confiança no dentista}

Péssima

Ruim

Regular

Boa

Excelente

Sem informação

Qualidade dos equipamentos odontológicos

Desatualizado

Moderno

Super moderno

Nem uma coisa nem outra

Sem informação

Qualidade de recursos

Sem informação

Não valeu a pena

Pouco valeu

Mais ou menos

Muito valeu

Até demais

Utilização de EPI e roupas limpas

Sem informação

Nunca

As vezes

Não lembra

Quase sempre

Sempre
18

101

8,5

88

47,9

41,7

1,9

1,9

19,9

42

54,0

45

21,3

2

0,9

$\begin{array}{cc}8 & 3,8 \\ 134 & 63,5 \\ 19 & 0,9 \\ 33 & 15,6 \\ 17 & 8,1\end{array}$

$\begin{array}{cc}3 & 1,4 \\ 1 & 0,5 \\ 4 & 1,9 \\ 40 & 19,0 \\ 143 & 67,8 \\ 20 & 9,5\end{array}$

$\begin{array}{cc}3 & 1,4 \\ 18 & 8,5 \\ 24 & 11,4 \\ 12 & 5,7 \\ 49 & 23,2 \\ 105 & 49,8\end{array}$

Fonte: Autores.

\subsection{Avaliação da equipe de saúde}

A Tabela 4 aponta os resultados das perguntas do questionário que diz respeito a opinião do paciente sobre os tratamentos recebidos pela equipe de saúde bucal. 
Tabela 4. Dados relacionados a opinião do paciente quanto ao tratamento recebido pela equipe de saúde dental. Patos, PB, 2020 .

\section{Procedimentos e relação paciente profissional}

Dor durante extração

Senti muito desconforto

Senti desconforto

Senti muito pouco desconforto

Total desconforto

Não fez procedimento de exodontia

Desconforto durante raspagem ou restauração

Senti muito desconforto

Senti desconforto

Senti muito pouco desconforto

48

34

104

Não realizou procedimentos de restauração ou raspagem

Restauração ficou arranhando após concluída

Não fez procedimentos de restauração

Senti total desconforto

Senti muito desconforto

Senti desconforto

Senti muito pouco desconforto

Não sentiu nada

Frequência dentista ensina sobre cuidados preventivos

Sem informação

Nunca

\section{Expectativas sobre o atendimento}

Sem informação

Nunca são atendidas

Diferentemente atendidas

Não lembro

Parcialmente atendidas

Igualmente atendidas 
Não me lembro 28

Na maioria das vezes me explica

Sempre me explica tudo

\section{Profissional costuma pedir opinião para melhor dia para} retorno

Nunca pede opinião

Raras vezes pede opinião

Não lembro

Na maioria das vezes pede opinião

Fonte: Autores.

\subsection{Análise bivariada}

Ao analisar a relação entre a localização das Unidades Básicas de Saúde (UBS) com a variável de acesso aos serviços odontológicos (vaga para o tratamento) não se observou relação estatisticamente significante ( $\mathrm{p}=0,081$ ), no entanto a localização da UBS apresentou relação significativa com a qualidade dos serviços (solução do problema) ( $\mathrm{p}=0,0015$ ), onde pôde-se perceber que 41,2\% (n=14) dos problemas não-resolvidos foram de pacientes da DGA 1.

Tabela 5. Associação entre as variáveis de acesso e qualidade dos serviços e a localização da UBS. Patos, 2020.

\begin{tabular}{|c|c|c|c|c|c|c|}
\hline & \multicolumn{3}{|c|}{ Localização da UBS } & \multirow[b]{2}{*}{ DGA4 } & \multirow[b]{2}{*}{ TOTAL } & \multirow[b]{2}{*}{ Valor de $\mathbf{p}^{(1)}$} \\
\hline & DGA1 & DGA2 & DGA3 & & & \\
\hline Dados de avaliação & $\mathrm{n}(\%)$ & $\mathrm{n}(\%)$ & $\mathrm{n}(\%)$ & & & \\
\hline \multicolumn{7}{|c|}{ SOLUÇÃO DO PROBLEMA } \\
\hline resolvido & $32(18,1)$ & $50(28,2)$ & $41(23,2)$ & $54(30,5)$ & $177(83,9)$ & 0,0015 \\
\hline \multicolumn{7}{|c|}{ VAGA PARA O TRATAMENTO } \\
\hline Difícil & $9(14,8)$ & $24(39,3)$ & $17(27,9)$ & $11(18,0)$ & $61(28,9)$ & 0,081 \\
\hline
\end{tabular}

Fonte: Autores.

\section{Discussão}

Os processos de avaliação dos serviços de saúde são de extrema importância, uma vez que os seus resultados são capazes de gerar novos conhecimentos, podendo assim fornecer subsídios para a estruturação de políticas públicas de saúde voltadas para atender a necessidade da população.

No Brasil, o Ministério da Saúde vem desenvolvendo, de forma mais sistemática, estratégias para avaliação e monitoramento da atenção primária em conjunto com as secretarias estaduais e municipais de saúde e instituições de ensino e pesquisa (Roncalli, 2010). Dentre os instrumentos utilizados pelo Ministério da Saúde (MS) para avaliar os serviços de saúde 
no Brasil destacam-se o PCA Tool - Brasil, a Avaliação Para Melhoria da Qualidade da Estratégia da Saúde da Família (AMQ) e os instrumentos que compõem o Programa Nacional de Melhoria do Acesso e da Qualidade na Atenção Básica (PMAQ - AB). Exclusivamente para os serviços odontológicos, Fernandes (2002) validou um Questionário de Avaliação de Serviços de Saúde Bucal - QASSaB, sendo amplamente utilizado quando se quer avaliar a qualidade dos serviços prestados na área de saúde bucal, sendo esse o questionário de escolha para realização desse estudo.

O município de Patos-PB está localizado no sertão da Paraíba, distante $307 \mathrm{Km}$ da capital João Pessoa, apresenta território de $512.719 \mathrm{~km}^{2}$, população estimada 105.531 habitantes e IDH 0,701 (alto) - PNUD/2010 (IBGE, 2013). O município conta com 41 Unidades Básicas de Saúde da Família (UBS)/Equipes de Saúde Bucal e 01 Centro de Especialidade Odontológicas (Brasil, 2021). Baseado nesses dados, há uma proporção de 01 UBS para cada 2.560 habitantes. Esses valores estão dentro do que é recomendado pelo Ministério da Saúde, por meio da Política Nacional da Atenção Básica (PNAB), onde cada Equipe de Saúde da Família deve ser responsável por uma cobertura populacional entre 2000 a 3500 pessoas, e tendo como referência para o cálculo do teto máximo de Equipes de Saúde da Família (ESF) com ou sem os profissionais de saúde bucal: população/2.400 (Brasil, 2017).

Santiago et al., (2012) realizou uma pesquisa com objetivo de avaliar a percepção dos usuários com a qualidade do atendimento nas Unidades de Saúde da Família do município de Recife-PE. Com a pesquisa foi possível notar que os usuários eram predominantemente adultos jovens, do sexo feminino, casados, com baixo nível de escolaridade e donas de casa. Nesse mesmo sentido, Guibu et al., (2017) observaram, ao avaliar o perfil de 8.676 usuários da atenção básica, que 75,8\% eram mulheres, a maioria de 18 a 39 anos de idade; 53,7\% com ensino fundamental e metade dos usuários foi classificada como classe C e $24,8 \%$ recebia bolsa-família. Nessa pesquisa pôde-se observar que a maioria dos usuários entrevistados eram mulheres, com idade entre 31-40 anos, casados, com renda mensal familiar de 1-3 salários mínimos, apresentando $2^{\circ}$ grau completo, moradores de casa própria e não beneficiário de programas sociais.

No presente estudo observou-se que os usuários se encontravam relativamente satisfeitos com o atendimento odontológico prestado na atenção básica da cidade de Patos-PB. Tendo sido relatado, pela maioria, que os problemas de saúde bucal foram resolvidos, num curto intervalo de tempo e sem dificuldade na marcação das consultas. Esses achados vão de encontro aos resultados de Moimaz, Rós, Bordin, Rovida e Garbin (2015) que também se propôs a avaliar a satisfação do usuário com o serviço público odontológico em um município de médio porte do estado de São Paulo (SP).

Nesse mesmo sentido, Campos et al., (2014) constatou que grande parte dos pacientes e usuários queixavam-se de demoras no atendimento, o que levavam muitos a chegarem às unidades básicas de saúde ainda de madrugada para conseguirem senha para atendimento, o que muitas vezes não garantia que chegariam a ter uma consulta médica ou um acolhimento inicial a suas queixas e, consequentemente, a não resolução dos seus problemas de saúde.

Shimizu e Ramos (2018) realizaram um estudo com o enfoque em avaliar a qualidade da Estratégia da Saúde da Família (ESF) do Distrito Federal na perspectiva dos usuários. Constataram em seu estudo que foram melhores avaliadas pelos usuários as dimensões sobre oferta de serviços, qualificação dos profissionais, qualidade da relação profissional e usuário e a continuidade do atendimento. Por outro lado, o enfoque familiar, enfoque comunitário e o acesso, principalmente, foram as que tiveram piores avaliação. Os achados relacionados ao acesso aos serviços divergem dos resultados dessa pesquisa, talvez seja pelo fato da cidade de Patos-PB ter uma boa cobertura populacional e, por esse motivo, as queixas dos usuários em relação ao acesso às UBSs sejam mínimas. No entanto no que concerne a avaliação da oferta dos serviços e a qualificação do profissional e relação profissional-paciente os achados de Shimizu e Ramos (2018) coincidem com o do presente estudo.

Pinho et al., (2020) avaliou o acesso e a acessibilidade às Unidades Básicas de Saúde nas diferentes regiões do Brasil caracterizando-o segundo os componentes organizacional e de infraestrutura. Em seu estudo pode-se constatar que $21,7 \%$ das Unidades Básicas de Saúde apresentava estrutura divergente do estabelecido pelo Ministério de Saúde e que existem unidades 
básicas que precisam ser reformuladas para garantir a melhoria do acesso. No estudo de Santiago et al., (2012) observaram que apesar da estratégia de saúde da família demonstrar melhora na eficácia e na qualidade dos serviços ainda é possível notar um número significativo de unidades com estruturas físicas inadequadas, não raramente, improvisadas. Tais achados não coincidem com os do presente estudo, uma vez que os itens relacionados à estrutura física das unidades de saúde, conforto, qualidade dos recursos e equipamentos foram todos, no geral, bem avaliados.

Barbosa Junior, Callefi e Chiroli (2018) constataram que grande parte do descontentamento dos pacientes com a UBS vinha em parte das condições de estrutura e equipamentos em sala de espera que afetava negativamente o conforto dos usuários, a desmotivação dos funcionários administrativos que refletia na relação dos usuários com os mesmos e dificuldades nos horários de atendimentos. Nesse estudo, a relação dos usuários com os funcionários, em grande parte, foi considerada boa ou excelente e os mesmos consideram também fácil ou muito fácil conseguir vaga para atendimento sem muito tempo de espera.

Quando os usuários foram indagados sobre a frequência com que os cirurgiões-dentistas orientam sobre cuidados preventivos em saúde bucal, observou-se que a maioria respondeu que "raramente" recebem essas informações. Sabe-se que uma das atribuições do cirurgião-dentista na atenção básica é realizar a atenção integral em saúde bucal (proteção da saúde, prevenção de agravos, diagnóstico, tratamento, reabilitação e manutenção da saúde) individual e coletiva, a todas as famílias (Brasil, 2008). Em relação às principais necessidades de tratamento relatadas pelos usuários, pôde-se observar que as exodontias e restaurações foram os procedimentos de maior procura. Tal fato demonstra que os pacientes, em sua maioria, procuram os serviços odontológicos apenas para procedimentos curativistas.

Echeverria, Dumith e Silva (2020) encontraram uma prevalência de dor de dente de $18 \%$ ao avaliarem 1.099 indivíduos no município de Rio Grande - RS. Nesse sentido, embora a maioria $(60,7 \%)$ dos participantes desse estudo relatarem nunca ter sentido dor de dente, ainda é alta a prevalência dessa patologia quando comparados ao estudo anterior.

A motivação para realização desse estudo se deu devido ao fato de que, no período de realização da pesquisa, a cidade de Patos-PB estava passando por uma instabilidade político-econômica muito grande, com alta rotatividade de gestores municipais e secretários, e embora o acesso e qualidade do atendimento odontológico da atenção básica de saúde da cidade tenha sido avaliado positivamente em quase todos os aspectos, a realidade da saúde pública do município vai de encontro com o que foi relatado pela maioria dos pacientes. Tal fato nos remete a possibilidade de que os pacientes se basearam em experiências positivas vivenciadas no passado e/ou não se sentiram confortáveis em relatar a realidade, já que as entrevistas aconteciam dentro dos estabelecimentos de saúde, ou mesmo, o bom relacionamento dos usuários com a equipe de saúde das UBSs pode ter influenciado nas avaliações.

\section{Conclusão}

Observou-se que os usuários das Unidades Básicas de Saúde de Patos-PB encontram-se relativamente satisfeitos com o atendimento odontológico realizado na atenção básica, tendo as suas queixas no geral prontamente atendidas pelos profissionais, com facilidade na marcação das consultas e resolução dos problemas. A maioria avaliou positivamente a estrutura física das unidades, bem como o relacionamento com a equipe de saúde. No entanto, maioria relatou que raramente recebe orientações preventivas relacionadas a saúde bucal e os procedimentos odontológicos de maior procura foram as exodontias e restaurações. Nesse sentido é necessário um maior incentivo à implementação de políticas públicas voltadas aos cuidados em saúde bucal nesse município.

\section{Referências}


Backes, D. S., Souza, M. H. T., Marchiori, M. T. C., Colome, J. S., Backes, M. T. S., \& Lunardi Filho, W. D. (2014). O Sistema Único de Saúde idealizado versus o realizado: contribuições da Enfermagem. Rev. Latino-Am. Enfermagem, 22 (6), 1026-1033.

Bahia, L. (2018). Trinta anos de Sistema Único de Saúde (SUS): uma transição necessária, mas insuficiente. Cadernos de Saúde Pública, 24 (7), 1-16.

Barbosa Junior, A. J., Callefi, M. H. B. M., \& Chiroli, D. M. G. (2018). Avaliação da qualidade do atendimento em uma unidade básica de saúde em Maringá/PR. Latin American Journal of Business Management, 9(1), 28-44.

Brandão, A. L. R. B. S., Giovanella, L., \& Campos, C. E. A. (2013). Avaliação da atenção básica pela perspectiva dos usuários: adaptação do instrumento EUROPEP para grandes centros urbanos brasileiros. Ciência \& Saúde Coletiva, 18(1), 103-114.

Brasil. (2008). Ministério da Saúde. Secretaria de Atenção à Saúde. Departamento de Atenção Básica. Saúde Bucal / Ministério da Saúde, Secretaria de Atenção à Saúde, Departamento de Atenção Básica.

Brasil. (2010). Ministério da Saúde. Secretaria de Atenção à Saúde. Política Nacional de Humanização. Formação e intervenção / Ministério da Saúde, Secretaria de Atenção à Saúde, Política Nacional de Humanização.

Brasil. (2010). Ministério da Saúde. Secretaria de Atenção em Saúde. Manual do instrumento de avaliação da atenção primária à saúde: primary care assessment tool pcatool.

Brasil. (2012). Ministério da Saúde. Secretaria de Atenção à Saúde. Departamento de Atenção Básica. Programa Nacional de Melhoria do Acesso e da Qualidade da Atenção Básica (PMAQ): manual instrutivo.

Brasil. (2017). Ministério da Saúde. Portaria n 2.436, de 21 de setembro de 2017. Aprova a Política Nacional de Atenção Básica, estabelecendo a revisão de diretrizes para a organização da Atenção Básica, no âmbito do Sistema Único de Saúde (SUS). http://www.bra-silsus.com.br/index.php/legislacoes/gabinete do-ministro/16247-portaria-n-2-436-de-21-de-setem $\neg$ bro-de-2017

Brasil. (2021). Ministério da Saúde. Banco de dados do Sistema Único de Saúde-DATASUS. http://www.datasus.gov.br

Brunhauser, A. L., Magro, M. L., \& Neves, M. (2013). Avaliação de serviço de saúde bucal: Um estudo comparativo. RFO UPF, 18(1), 24-31.

Campos, R. T. O., Ferrer, A. L., Gama, C. A. P., Campos, G. W. S., Trapé, T. L., \& Dantas, D. V. (2014). Avaliação da qualidade do acesso na atenção primária de uma grande cidade brasileira na perspectiva dos usuários. Saúde Debate, 38, 252-264.

Echeverria, M. S., Dumith, S. C., \& Silva, A. E. R. (2020). Prevalência e fatores associados a dor dentária - estudo de base populacional com adultos e idosos do sul do Brasil. Revista de Odontologia da UNESP, 49:e20200039, 1-9.

Fernandes, L. M. A. G. (2002). Validação de um instrumento para avaliação da satisfação dos usuários, com os serviços públicos de saúde bucal. Dissertação de doutorado, Faculdade de Odontologia do Pernambuco, Camaragibe, PE, Brasil.

Gomide, M. F. S., Pinto, I. C., Bulgarelli, A. F., Santos, A. L. P., \& Gallardo, M. P. S. (2018). User satisfaction with primary health care: an analysis of access and care. Interface, 22(68), 387-398.

Guibu, I. A., Moraes, J. C., Guerra Junior, A. A., Costa, E. A., Acurcio, F. A., Costa, K. S., Karnikowski, M. G. O., Soeiro, O. M., Leite, S. N., \& Alvares, J. (2017). Características principais dos usuários dos serviços de atenção primária à saúde no Brasil. Revista de Saúde Pública, 51, 1-13.

Instituto Brasileiro de Geografia e Estatística. (2000). Acesso e utilização de serviços de saúde: PNAD 1998. Rio de Janeiro: IBGE.

Magalhães, B. G., Oliveira, R. S., Goés, P. S. A., \& Figueiredo, N. (2015). Avaliação da qualidade dos serviços prestados pelos Centros de Especialidade Odontológicas: Visão dos usuários. Cad. Saúde Colet, 23 (1), 76-85.

Moimaz, S. A. S., Rós, D. T., Bordin, D., Rovida, T. A. S., \& Garbin, C. A.S. (2015). Satisfação e perfil de usuários do serviço odontológico no Sistema Único de Saúde. Rev. Odonto, 20(3), 334-339.

Moura, D. C. N., Pinto, J. R., \& Aragão, A. E. A. (2016). Perfil dos profissionais atuantes na gestão em saúde frente ao novo modelo de reorganização do SUS: a regionalização. Tempus - Actas De Saúde Coletiva, 10(1), 75-93.

Pereira, A. S., Shitsuka, D. M., Parreira, F. J., \& Shitsuka, R. 2018. Metodologia da pesquisa científica. Consultado em 10 de jul. de 2021. Disponível em https://repositorio.ufsm.br/bitstream/handle/1/15824/Lic_Computacao_Metodologia-Pesquisa-Cientifica.pdf?sequence=1

Perez, L. G., Sheridan, J. D., Nicholls, A. Y., Mues, K. E., Saleme, P. S., Resende, J. C., Ferreira, J. A. G., \& Leon, J. S. (2013). Professional and community satisfaction with the Brazilian family health strategy. Rev. Saúde Públ, 47(2), 403-413.

Pinho, E. C. C., Cunha, T. A. N., Lemos, M., Ferreira, G. R. O. N., Lourenção, L. G., Pinheiro, H. H. C., Botelho, E. P., \& Cunha, C. L. F. (2020). Acesso e acessibilidade na atenção primária à saúde no Brasil. Enfermagem em Foco, 11 (2), 168-175.

Santiago, R. F., Mendes, A. C. G., Miranda, G. M. D., Duarte, P. O., Furtado, B. M. A. S. M., \& Souza, W. V. (2013). Qualidade do atendimento nas Unidades de Saúde da Família no município de Recife: a percepção dos usuários. Ciência \& Saúde Coletiva, 18(1), 35-44.

Shimizu, H. E., \& Ramos, M. C. (2019). Evaluation of quality of the family health strategy in the Federal District. Revista Brasileira de Enfermagem, 72 (2), 367-374.

Todos os autores participaram de todas as etapas da pesquisa e tiveram igual contribuição na execução do projeto e redação do artigo. 